\title{
The evolution of tubular structures in a viscous quark gluon plasma
}

\author{
F. S. Navarra*t \\ Instituto de Física, Universidade de São Paulo \\ E-mail: navarraeif.usp.br
}

\section{D.A. Fogaça}

Instituto de Física, Universidade de São Paulo

E-mail: davideif.usp.br

\section{L.G. Ferreira Filho}

Faculdade de Tecnologia, Universidade do Estado do Rio de Janeiro

E-mail: gonzaga@fat.uerj.br

\begin{abstract}
We study the radial expansion of cylindrical tubes in a hot QGP described by the equation of state of the MIT bag model. These tubes are treated as perturbations in the energy density of the system which is formed in heavy ion collisions at RHIC and LHC. We start from the equations of viscous relativistic hydrodynamics in two spatial dimensions with cylindrical symmetry and perform an expansion of these equations in a small parameter, conserving the nonlinearity of the hydrodynamical formalism. The relativistic viscous fluid is studied with a relativistic NavierStokes equation and the perturbations are governed by the Burgers equation. We estimate the typical expansion time of the tubes.
\end{abstract}

Sixth International Conference on Quarks and Nuclear Physics

April 16-20, 2012

Ecole Polytechnique, Palaiseau, Paris

\footnotetext{
* Speaker.

${ }^{\dagger}$ The authors are grateful to R. Venugopalan, R.P.G. Andrade, S.B. Duarte, J. Noronha and M. Strickland for enlightening discussions. This work was partially financed by the Brazilian funding agencies CAPES, CNPq and FAPESP.
} 


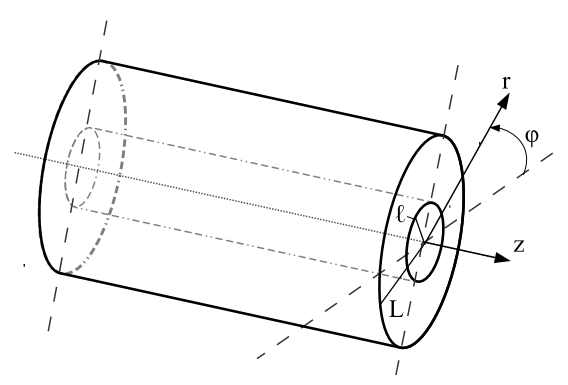

Figure 1: Tubular perturbations on a QGP background. The inner cylinder of radius $l$ represents a tube of energy density higher than the background, shown as a cylindrical fireball of radius $L$. The perturbation expands radially.

\section{Introduction}

Theoretical works and also the analysis of experimental data on relativistic heavy ion collisions suggest that in the early times of these collisions color flux tubes are formed. Hydrodynamical studies [1,2] indicate that some experimental features observed at RHIC and LHC can be understood if it is assumed that these tubes survive the thermalization stage and form "tubular" structures that persist for some time during the hydrodynamical expansion in the thermalized quark-gluon plasma (QGP). In [1, 2] it has been argued that these structures may have a common hydrodynamic origin: the combined effect of longitudinal high energy density tubes (leftover from initial particle collisions) and transverse expansion. Such tubular structures, which are nearly uniform in the longitudinal direction, may be considered as cylindrical perturbations in the energy density upon a continuous background as depicted in Fig. 1. The propagation of perturbations on the top of QGP background has been investigated in several works $[3,4,5,6]$. In most of these works [3, 4] a linearized version of hydrodynamics is employed. We have tried to keep the nonlinear terms in the equations which describe the evolution of the perturbations [5, 6]. This extends the validity of our formalism to perturbations which are not so small. In this work we study how fast the tubes expand in the viscous QGP. We write the relativistic viscous hydrodynamical equations for the propagation of cylindrical perturbations along the radial direction (see Fig. 1). We obtain a non-linear wave and solve it numerically to estimate the time needed for a tube of initial radius of the order of $1 \mathrm{fm}$ to grow and reach the typical radius of the system formed in heavy ion collisions, which is of the order of $7 \mathrm{fm}$. If the tube expansion time were much shorter than the lifetime of the fireball, then the tube would be very rapidly incorporated in the fireball and it would produce no visible effect in the final state particle correlation measurements. It is well known that the relativistic version of the Navier-Stokes equation does not constitute a causal theory. We strongly recommend the reading of [7] to understand the subject in details. Besides these conceptual issues of stability and causality we perform a Navier-Stokes approach without worrying about microscopic time scales, due to the nonlinear expansion as seen in [8]. A future and more complete version of this work in relativistic viscous hydrodynamics is in progress with the use of Müller-Israel-Stewart theory, which is a causal theory [7]. Due to dissipation, viscosity damps the perturbations, which are then more easily mixed with the background fluid, loosing their influence on final state particle correlations. 


\section{Relativistic viscous Fluid Dynamics}

A review text on relativistic viscous hydrodynamics is [7]. Approximation schemes which conserve nonlinearities can be found in [9] and their application to the study of nonlinear waves in cold and warm nuclear matter can be found in $[8,10]$ and references therein. Throughout this work we use $c=1, \hbar=1$ and the Boltzmann constant $k_{B}=1$. We start our discussion considering two coaxial cylinders. The inner and narrower cylinder represents the flux tube which is a perturbation in energy density $\varepsilon$. The outer and larger cylinder represents the fireball with a uniform energy density $\varepsilon_{0}\left(\varepsilon_{0} \leq \varepsilon\right)$. We will study the expansion of the flux tube in the center of mass system of the fireball. It is natural to choose spatial cylindrical coordinates $(z, r, \phi)$. The velocity four-vector $u^{v}$ is defined as $u^{0}=\gamma, \vec{u}=\gamma \vec{v}$, where $\gamma$ is the Lorentz factor $\gamma=\left(1-v^{2}\right)^{-1 / 2}$ and thus $u^{v} u_{v}=1$. The velocity field of matter is given by $\vec{v}=\vec{v}(t, r, z, \phi)$. Because of the azimuthal symmetry we do not have components along the $\phi$ direction and consequently no terms involving $\partial / \partial \phi$ will survive in what follows. The details of viscous hydrodynamics are given in $[7,8]$. We shall use the basic equations for this work, which are the relativistic version of the Navier-Stokes equation:

$$
\begin{gathered}
(\varepsilon+p) \gamma^{2}\left(\frac{\partial}{\partial t}+\vec{v} \cdot \vec{\nabla}\right) \vec{v}+\vec{v} \frac{\partial p}{\partial t}+\vec{\nabla} p-\eta \vec{v}\left\{\partial_{\mu} \partial^{\mu} \gamma+\partial_{\mu} \frac{\partial u^{\mu}}{\partial t}-\partial_{\mu}\left[\gamma\left(\frac{\partial}{\partial t}+\vec{v} \cdot \vec{\nabla}\right)\left(\gamma u^{\mu}\right)\right]\right\} \\
-\vec{v}\left(\zeta-\frac{2}{3} \eta\right) \frac{\partial}{\partial t}\left[\frac{\partial \gamma}{\partial t}+\vec{\nabla} \cdot(\gamma \vec{v})\right]+\vec{v}\left(\zeta-\frac{2}{3} \eta\right) \partial_{\mu}\left\{\gamma u^{\mu}\left[\frac{\partial \gamma}{\partial t}+\vec{\nabla} \cdot(\gamma \vec{v})\right]\right\} \\
+\eta\left\{\partial_{\mu} \partial^{\mu}(\gamma \vec{v})-\partial_{\mu} \vec{\nabla} u^{\mu}-\partial_{\mu}\left[\gamma\left(\frac{\partial}{\partial t}+\vec{v} \cdot \vec{\nabla}\right)\left(\gamma \vec{v} u^{\mu}\right)\right]\right\}-\left(\zeta-\frac{2}{3} \eta\right) \vec{\nabla}\left[\frac{\partial \gamma}{\partial t}+\vec{\nabla} \cdot(\gamma \vec{v})\right] \\
-\left(\zeta-\frac{2}{3} \eta\right) \partial_{\mu}\left\{\gamma \vec{v} u^{\mu}\left[\frac{\partial \gamma}{\partial t}+\vec{\nabla} \cdot(\gamma \vec{v})\right]\right\}=0
\end{gathered}
$$

and the relativistic version of the continuity equation for the entropy density $s$ :

$$
\begin{gathered}
\gamma \frac{\partial s}{\partial t}+\gamma \vec{\nabla} s \cdot \vec{v}+s \frac{\partial \gamma}{\partial t}+s \vec{\nabla} \gamma \cdot \vec{v}+\gamma s \vec{\nabla} \cdot \vec{v}=-\frac{\eta}{T}\left(\frac{\partial \gamma}{\partial t}\right)^{2}-2 \frac{\eta}{T}\left[\vec{\nabla} \gamma \cdot \frac{\partial}{\partial t}(\gamma \vec{v})\right] \\
-\frac{\eta}{T}\left(\partial^{i} u^{j}\right) \partial_{j} u_{i}+\frac{1}{T}\left(\frac{2}{3} \eta+\zeta\right)\left[\frac{\partial \gamma}{\partial t}+\gamma \vec{\nabla} \cdot \vec{v}+\vec{\nabla} \gamma \cdot \vec{v}\right]^{2}
\end{gathered}
$$

where $\eta$ is the shear viscosity coefficient and $\zeta$ is the bulk viscosity coefficient. The ideal fluid is recovered when $\eta=\zeta=0[7,8]$.

\section{Equation of state}

From the thermodynamics of the MIT bag model we have (see details in $[5,8]$ ) the speed of sound $c_{s}$, given by $c_{s}^{2}=1 / 3$, the bag constant $\mathscr{B}$, given by $\mathscr{B}=37 \pi^{2}\left(T_{B}\right)^{4} / 30$, which is chosen to be $\mathscr{B}^{1 / 4}=170 \mathrm{MeV}\left(T_{B}=91 \mathrm{MeV}\right)$ and the relations:

$$
s=s(\varepsilon)=4 \frac{37}{90} \pi^{2}\left[\frac{30}{37 \pi^{2}}(\varepsilon-\mathscr{B})\right]^{3 / 4}, \quad \varepsilon+p=\frac{148}{90} \pi^{2} T^{4}, \quad \vec{\nabla} p=\frac{1}{3} \vec{\nabla} \varepsilon \quad \text { and } \quad \frac{\partial p}{\partial t}=\frac{1}{3} \frac{\partial \varepsilon}{\partial t}
$$




\section{The non-linear wave equation}

We follow the procedure described in [8] combining the equations (2.1) and (2.2) and using (3.1) to obtain the non-linear wave equation:

$$
\frac{\partial}{\partial r}\left\{\frac{\partial \hat{\varepsilon}_{1}}{\partial t}+c_{s} \frac{\partial \hat{\varepsilon}_{1}}{\partial r}+\frac{c_{s}}{2}\left[1+\left(\frac{T_{B}}{T_{0}}\right)^{4}\right] \hat{\varepsilon}_{1} \frac{\partial \hat{\varepsilon}_{1}}{\partial r}+\frac{\hat{\varepsilon}_{1}}{2 t}-\frac{1}{T_{0}}\left(\frac{\zeta}{s}+\frac{4}{3} \frac{\eta}{s}\right) \frac{\partial^{2} \hat{\varepsilon}_{1}}{\partial r^{2}}\right\}+\frac{c_{s}}{2} \frac{\partial^{2} \hat{\varepsilon}_{1}}{\partial z^{2}}=0
$$

for the dimensionless small perturbation in the energy density $\hat{\varepsilon}_{1} \equiv \sigma \varepsilon_{1}$, which comes from the expansion $\hat{\varepsilon}=\varepsilon / \varepsilon_{0}=1+\sigma \varepsilon_{1}+\sigma^{2} \varepsilon_{2}+\sigma^{3} \varepsilon_{3}+\ldots$ where $\sigma$ is a small parameter [9] and $\varepsilon_{0}$ is the energy density of the background. We have also the dimensionless ratios $\eta / s$ and $\zeta / s$, which are well studied in the literature $[11,12]$.

\section{Numerical results and discussion}

For simplicity, we assume that when they are formed and also throughout the expansion the tubes are uniform along the longitudinal direction and therefore $\partial^{2} \hat{\varepsilon}_{1} / \partial z^{2}=0$. Integrating (4.1) with respect to $r$ and setting the integration constant to zero we arrive at the famous cylindrical Burgers equation [13] for the viscous fluid:

$$
\frac{\partial \hat{\varepsilon}_{1}}{\partial t}+c_{s} \frac{\partial \hat{\varepsilon}_{1}}{\partial r}+\frac{c_{s}}{2}\left[1+\left(\frac{T_{B}}{T_{0}}\right)^{4}\right] \hat{\varepsilon}_{1} \frac{\partial \hat{\varepsilon}_{1}}{\partial r}+\frac{\hat{\varepsilon}_{1}}{2 t}=\frac{1}{T_{0}}\left(\frac{\zeta}{s}+\frac{4}{3} \frac{\eta}{s}\right) \frac{\partial^{2} \hat{\varepsilon}_{1}}{\partial r^{2}}
$$

The initial condition is given by a gaussian pulse in $\hat{\varepsilon}_{1}$ :

$$
\hat{\varepsilon}_{1}=A e^{-r^{2} / r_{0}^{2}}
$$

where the amplitude $A<1$ and the approximate width $r_{0}$ are parameters which depend on the dynamics of flux tube formation. For simplicity we shall refer to $r_{0}$ as the initial "radius" of the tube. According to current estimates [12] the transverse size of the tubes is of the order of $1 \mathrm{fm}$ and thus in our calculations $r_{0}=0.1 \mathrm{fm}$ and $r_{0}=0.8 \mathrm{fm}$. We consider hot QGP at temperatures $T_{0}=150 \mathrm{MeV}$ and $T_{0}=500 \mathrm{MeV}$ treated as a viscous fluid $(\eta / s=0.08$ and $\zeta / s=0)[11,12]$. The strong influence of viscosity can be seen most clearly in the comparison between Fig. 2a) and 2b). Viscosity damps the amplitude of the pulse by a factor ten in $1 \mathrm{fm}$ ! Increasing the temperature of the medium reduces the effect of viscosity as it can be seen from the factor $1 / T_{0}$ in (5.1). However the comparison between Fig. 2b) and 2d) shows that this reduction is not very strong. The role played by viscosity is also reduced when the initial radius parameter of the tube goes from $r_{0}=0.1$ to $0.8 \mathrm{fm}$. This is easy to understand looking at (5.2) and then at the right hand side of (5.1). A broader initial distribution generates smaller spatial gradients appearing in the viscosity term of (5.1), which becomes smaller. If we also increase the initial amplitude from $A=0.5$ (Fig. 2) to $A=0.8$ (Fig. 3), the relevance of viscosity remains the same.

The introduction of viscosity in our calculations is what more strongly changes them. Due to dissipation, viscosity strongly damps and broadens the tubes during their expansion and they are more easily mixed with the background fluid, loosing their influence on final state particle correlations. This is a robust conclusion of our numerical analysis since it remains valid in all 
situations considered. Moreover viscosity prevents the perturbation wave from breaking, as can be seen comparing, for example, Fig. 3a) with 3b). Looking at the time evolution of the peaks of the pulses, we can have an idea of the velocity with which they propagate. Comparing the left with right side of all figures we can see the velocity of the pulses is only weakly changed by viscosity. This velocity is defined by the sound velocity, which in our approach is the same both for ideal and viscous fluids. An important conclusion of our work is that viscosity strongly affects the propagation of perturbations in the quark gluon plasma. This conclusion was obtained with the relativistic Navier-Stokes formalism and it would be interesting to check if it remains valid in other relativistic theories of viscosity.

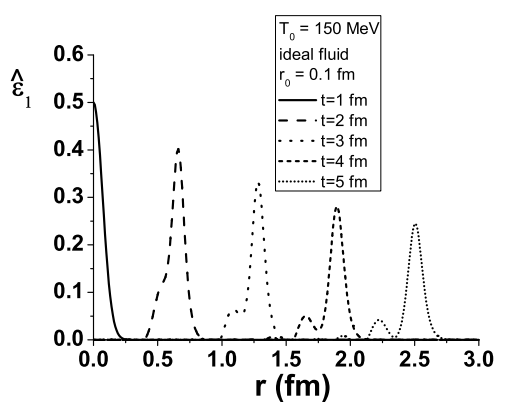

(a)

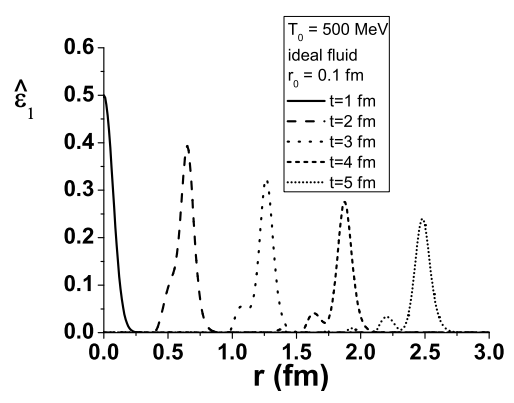

(c)

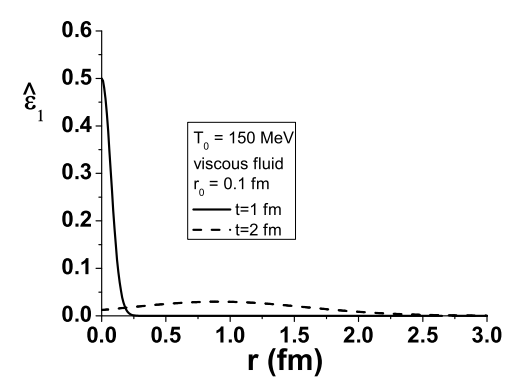

(b)

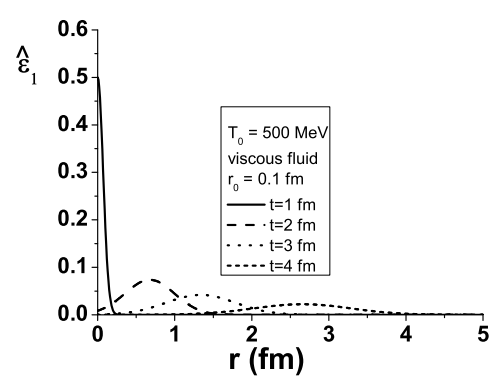

(d)

Figure 2: Numerical solutions of Burgers equation for an ideal fluid (left) and for a viscous fluid (right) with initial amplitude $A=0.5$.

\section{References}

[1] Y. Hama, R.P.G. Andrade, F. Grassi, W. -L. Qian, Nonlin. Phenom. Complex Syst. 12 , 466 (2009); R.P.G. Andrade, F. Grassi, Y. Hama and W.-L. Qian, arXiv:1012.5275 [hep-ph]; J. Phys. G G 37, 094043 (2010); R.P.G. Andrade, F. Gardim, F. Grassi, Y. Hama and W.L. Qian, J. Phys. G 38, 124123 (2011).

[2] R.P.G. Andrade, F. Grassi, Y. Hama and W.L. Qian, Nucl. Phys. A 854, 81 (2011); arXiv:1008.4612 [nucl-th].

[3] P. Staig and E. Shuryak, arXiv:1109.6633 [nucl-th]. 


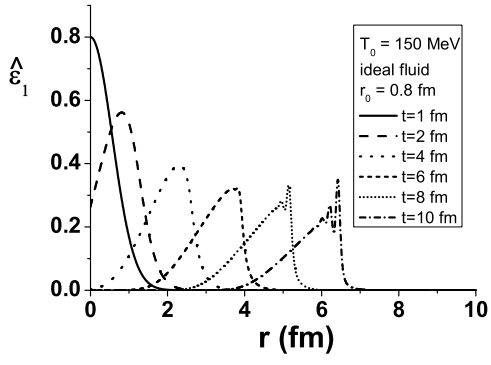

(a)

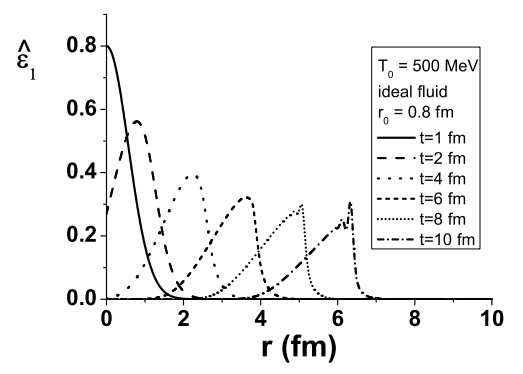

(c)

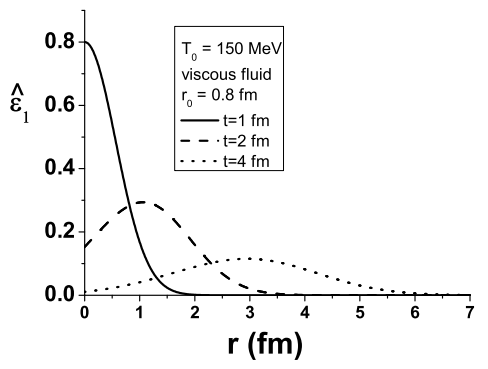

(b)

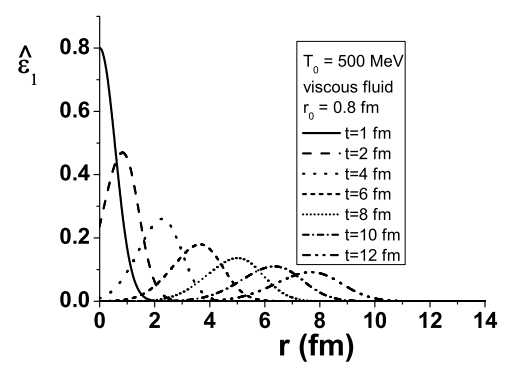

(d)

Figure 3: Numerical solutions of Burgers equation for an ideal fluid (left) and for a viscous fluid (right) with initial amplitude $A=0.8$.

[4] P. Staig and E. Shuryak, Phys. Rev. C 84, 044912 (2011); Phys. Rev. C 84, 034908 (2011); arXiv:1106.3243.

[5] D. A. Fogaça, L. G. Ferreira Filho and F. S. Navarra, Phys. Rev. C 81, 055211 (2010).

[6] D. A. Fogaca, F. S. Navarra and L. G. Ferreira Filho, Phys. Rev. D 84, 054011 (2011).

[7] P. Romatschke, Int. J. Mod. Phys. E 19, 1 (2010).

[8] D. A. Fogaça, F.S. Navarra and L. G. Ferreira Filho, Nucl. Phys. A 887, 22 (2012).

[9] R. C. Davidson, "Methods in Nonlinear Plasma Theory", Academic Press, New York an London, 1972, pages 20 and 21.

[10] D.A. Fogaça and F.S. Navarra, Phys. Lett. B 639, 629 (2006); D.A. Fogaça and F.S. Navarra, Phys. Lett. B 645, 408 (2007); D.A. Fogaça and F.S. Navarra, Nucl. Phys. A 790, 619c (2007); Int. J. Mod. Phys. E 16, 3019 (2007); D.A. Fogaça, L. G. Ferreira Filho and F.S. Navarra, Nucl. Phys. A 819, 150 (2009).

[11] See, for example, A.K. Chaudhuri, arXiv:1111.5713 [nucl-th] and also Victor Roy and A.K. Chaudhuri, arXiv:1201.4230v1 [nucl-th].

[12] B. Schenke, P. Tribedy and R. Venugopalan, arXiv:1202.6646 [nucl-th].

[13] A. A. Mamun and P. K. Shukla, Europhys. Lett. 87, 25001 (2009); A. A. Mamun and P. K. Shukla, Europhys. Lett. 94, 65002 (2011); B. Sahu, Bulg. J. Phys. 38, 175 (2011). 